\title{
The role of claudin-5 in blood-brain barrier (BBB) and brain metastases (Review)
}

\author{
WANG JIA ${ }^{1}$, RUNCHUN LU ${ }^{1}$, TRACEY A. MARTIN ${ }^{2}$ and WEN G. JIANG ${ }^{2}$ \\ ${ }^{1}$ Department of Neurosurgery, Beijing Tiantan Hospital, Capital Medical University, Beijing 100050, \\ P.R. China; ${ }^{2}$ Cardiff University-Capital Medical University Joint Centre for Biomedical Research, \\ Cardiff University School of Medicine, Cardiff CF14 4XN, UK
}

Received April 26, 2013; Accepted November 19, 2013

DOI: $10.3892 / \mathrm{mmr} .2013 .1875$

\begin{abstract}
Metastatic brain tumours are frequently observed in patients with lung, breast and malignant melanoma and a severe complication of metastatic cancers. With improved primary cancer treatments, including surgery, radiation therapy and chemotherapy, patients are now living longer following initial treatment, compared with previous treatments. Brain metastasis (BM) remains a significant clinical issue. Since BM represents a major therapeutic challenge, it is vital that the mechanisms of interaction between tumour cells and the blood-brain barrier (BBB), as well as the method by which tumour cells establish metastatic tumours in the brain, are understood. A key step in BM is the interaction and penetration of the BBB by cancer cells. The BBB consists of endothelial cells, pericytes, astrocytes and a number of molecular structures between these cells. The BBB relies on the tight junctions (TJs) that are present between the endothelial cells of the brain capillaries to provide a closed environment for the brain. TJs comprise a number of proteins, including occludin, claudins and junctional adhesion molecules (JAMs). Among them, claudins are the key integral proteins that regulate BBB permeability. It has previously been shown that claudin-5, not only regulates paracellular ionic selectivity, but also plays a role in the regulation of tumour cell motility, suggesting that TJs and claudin-5 contribute to the control of BM. This study reviews the role of claudin-5 in the regulation of BBB permeability during the brain metastatic process.
\end{abstract}

Correspondence to: Dr Wang Jia, Department of Neurosurgery, Beijing Tiantan Hospital, Capital Medical University, No. 6 TianTan Xili, Beijing 100050, P.R. China

E-mail: jwttyy@sina.com

Professor Wen G. Jiang, Cardiff University-Capital Medical University Joint Centre for Biomedical Research, Cardiff University School of Medicine, Henry Wellcome Building, Heath Park, Cardiff CF14 4XN, UK

E-mail: jiangw@cf.ac.uk

Key words: blood brain barrier, brain metastases, tight junctions, claudins, claudin-5, permeability

\section{Contents}

1. Brain metastases (BM)

2. Blood-brain barrier (BBB)

3. Composition of tight junctions (TJs) at the BBB

4. Claudins

5. Claudin-5 plays a role in the process of BM via the regulation of BBB permeability

6. Conclusion and future prospects

\section{BM}

$\mathrm{BM}$ are the most life-threatening complications of cancer, the exact prevalence of which is not clearly known. The incidence of BM in the US population is $\sim 170,000$ new cases/year, with a prevalence of $8.3 / 100,000(1,2)$. Clinical studies have shown that $\sim 8.5 \%$ of cancer patients present with BM, but a biopsy study shows a significantly higher BM incidence rate of $8.7-26 \%$ in a cohort of patients with carcinoma $(3,4)$. The majority of studies have demonstrated that the incidence of $\mathrm{BM}$ is equal to or 2-10 times that of the primary intracranial tumours (1-6). Incidence has increased with the availability of improved neuroimaging techniques that aid in the early diagnosis and effective systemic treatment regimens, leading to a prolonged life, thus allowing cancer to disseminate to the brain.

The primary tumours most likely to metastasize to the brain are located in the lung (50\%), breast (15-20\%), skin (melanoma) (10-15\%), colon-rectum (2-12\%), kidney (1-8\%) and thyroid gland (1-10\%). The primary site is unknown in $<10-15 \%$ of the patients. Soft tissue sarcoma, childhood Ewing sarcoma and childhood rhabdomyosarcoma are also significant sources of brain metastases $(4,7)$. Among these tumours, melanoma metastasizes to the brain with one of the highest frequencies. However, the most common sources of BM in children are osteogenic sarcoma, soft tissue sarcoma and germ cell tumours, which is different from those of adults (8).

Metastatic tumours most commonly invade the cerebral hemispheres (80\%), cerebellum (15\%) and brainstem $(<5 \%)(7,9,10)$. This distribution is hypothesized to reflect the correlation between the distribution of tumours and cerebral vasculature. The mean age of presentation for brain metastases 
is 55-65 years. The clinical manifestation of BM varies with the location of the metastatic tumours. The most common symptom is headache, occurring in $24-53 \%$ of patients. This is followed by limb weaknesses, altered neurocognitive function, seizures and ataxia (7). The majority (70\%) of patients with BM present with multiple lesions and only $30 \%$ present with a solitary lesion (8).

Comprehensive treatment for BM includes whole-brain radiation therapy, stereotactic radiosurgery, radiosensitizers, chemotherapy and surgery (7-9,11-14). Targeted therapies are providing promising results $(5,15)$. The median survival time from time of diagnosis is only 1 month for de novo patients (7). Patients receiving whole-brain radiation therapy have a median survival of 4-5 months, while patients treated with multidisciplinary management have a survival time of $\leq 12$ months (16). Since brain metastases represent a significant therapeutic challenge, it is necessary to understand the mechanisms by which tumour cells interact with the BBB in order to determine targets of prevention and treatment of BM formation.

The formation of BM is a complex series of processes in which detached primary tumour cells are transported through blood vessels, adhere to vascular endothelial cells, transmigrate through the BBB into brain parenchyma where they lodge and proliferate in the new location to form a secondary tumour mass $(3,5,9)$. A number of key molecules and proteins regulate this process. The only route for BM is through the blood vessels, due to the lack of lymphatic duct in the central nervous system (CNS). Regardless of their source, metastatic tumour cells are capable of transmigrating through the $\mathrm{BBB}$ to invade the brain.

\section{BBB}

The BBB is a barrier between blood circulation and brain parenchyma, providing anatomical and physiological protection for the CNS, supplying brain tissue with nutrients, filtering harmful compounds from the brain back to the bloodstream and shielding the brain from toxic substances in the blood. The BBB consists of four primary cellular elements: cerebral endothelial cells (ECs), astrocyte end-feet, microglial cells and pericytes (17).

ECs lining the brain capillaries are thin, flat cells interconnected by TJs. Ultrastructure studies (17-20) demonstrated that these ECs have the following characteristics: ECs forming TJs at their adjacent margins, produced by the interaction of a number of transmembrane proteins projecting into the paracellular space and effectively sealing it, thus confining penetration across brain endothelium to transcellular mechanisms; endothelial cytoplasm lacking fenestrations typically present in peripheral-tissue capillaries; fewer pinocytotic vesicles compared with peripheral endothelial cells and more mitochondria, suggesting important metabolic activity (17-22). Cerebral endothelial cells share common features with other endothelia, including the presence of factor VIII, high alkaline phosphatase and $\gamma$-glutamyl transpeptidase activity, uptake of acetylated low-density lipoprotein and epithelia, high transendothelial electrical resistance (TEER), a continuous line of tight junctions and low level of pinocytosis, the latter being indispensable for the barrier function (11).

Pericytes are located in the duplication of the basement membrane, in close contact with endothelial cells.
Gap junctions have been described between the two cell types (23). Pericyte-endothelial cell interactions were observed as being significant in the following areas: angiogenesis, BBB formation and maintenance, vascular stability and angioarchitecture, regulation of capillary blood flow and clearance of toxic cellular byproducts necessary for proper CNS homeostasis and neuronal function (24). Defects or absence of pericytes may lead to a number of CNS diseases, including neurodegeneration and neurovascular diseases and injury (25). Astrocytic end-feet almost completely ensheath the capillary walls, thereby covering endothelial cells, as well as the intimately associated pericytes (26). The coverage is not complete, allowing a direct contact of nerve endings with the basal membrane $(27,28)$. Astrocytes are associated with BBB permeability and ionic and water regulation.

Microglia are another type of cell in close contact with cerebral vessels. Microglia are primary immune effector cells in the brain and spinal cord and are important in neuroinflammatory processes (29). These cells provide immune surveillance and are mobilized in response to disparate diseases and injuries. They are also essential defenders against a number of neurodegenerative diseases $(29,30)$. However, the exact role of microglia in the neurovascular unit remains poorly understood and occasionally controversial.

In the human brain, neurons are generally not in direct contact with cerebral endothelial cells. It is currently unclear as to whether there are signals from endothelial cells to neurons and vice versa, which may be significant for brain homeostasis or neuronal function. The presence of neurotransmitter receptors on endothelial cells was observed in laboratory rats, suggesting a communication between the two cells.

Aside from these cells, there is a specialized extracellular matrix, the basement membrane, covering endothelial cells from the outside astrocytes and pericytes. Its primary protein components include collagen, particularly type IV, fibronectin, laminin, tenascin and proteoglycans. The extracellular matrix serves as an anchor for endothelial cells and modulates TJ protein expression. In addition, it is involved in the alteration in $\mathrm{BBB}$ permeability during the pathological process of brain tumour and cerebral ischemia $(31,32)$. All the aforementioned components form the BBB which acts extremely effectively to: i) maintain the ionic composition optimal for synaptic signaling function; ii) protect the brain from neurotransmitters in the rest of the body; iii) prevent macromolecules from entering the brain; iv) shield the CNS from neurotoxins circulating in the blood and v) ensure an adequate brain nutrition supply. Thus, the BBB provides a stable microenvironment that is critical for complex neural function and protects the CNS from chemical insult and damage (33). Transport across the brain endothelium is strictly limited through a 4-fold defense line: paracellular barrier, represented by interendothelial junctions; transcellular barrier, assured by the low level of endocytosis and transcytosis; enzymatic barrier, including acetylcholinesterase, alkaline phosphatase, $\gamma$-glutamyl transpeptidase, monoamine oxidases and drug-metabolizing enzymes and the efflux transporters, ABC-B1, -C1, -C4, -C5 and -G2 (34). Small gaseous molecules, including $\mathrm{O}_{2}$ and $\mathrm{CO}_{2}$, freely diffuse through the lipid membranes which serves as a route of entry for small lipophilic agents, including barbiturates, nicotine and ethanol. However, specific blood-to-brain influx transport 
systems exist to supply nutrients, including glucose, amino acids and nucleotides, which cannot freely diffuse to the brain.

TJs constitute the primary structure of the paracellular barrier between endothelial cells and are responsible for regulating BBB permeability.

\section{Composition of TJs at BBB}

TJs of the BBB are present between the endothelial cells of brain capillaries. There are two primary classes of proteins at the TJs: i) transmembrane proteins, including occludin, claudins and junctional adhesion molecules (JAMs); ii) peripheral proteins: the zonula occludens family, AF6/afadin, multi-PDZ domain protein 1 (MUPP1), membrane-associated guanylate kinase inverted (MAGI)-1, -2 and -3 , PAR-3 and -6 , and heterotrimeric G-proteins.

Occludin, the first identified transmembrane TJ protein (35), is a 60-65 kDa molecule. It is characterized by four transmembrane regions, two extracellular loops, a shorter N-terminal and a longer $\mathrm{C}$-terminal cytoplasmic domain. The $\mathrm{N}$ - and $\mathrm{C}$-terminus are intracellular. The exact role of occludin in TJs remains unknown, however, the barrier function by TJs remains present in the intestinal epithelium of occludin knock-out mice, suggesting that occludin is likely a structural component of TJs. An increasing number of studies indicate that occludin is important in the formation of TJs $(36,37)$. Occludin function is regulated by GTPases, proteases and cytokines (38).

JAMs are members of an immunoglobulin subfamily, with a molecular weight of $\sim 40 \mathrm{kDa}$. JAM comprises three structural domains: an extracellular domain with two immunoglobulin-like loops, a single transmembrane domain and a short intracellular domain. The JAM family of proteins are divided into two groups based on their sequence similarities: the closely related JAM-A, -B and -C and the more distantly related coxackie and adenorevirus (CAR), CAR-like membrane protein, endothelial cell-selective adhesion molecule (ESAM) and JAM-4 (39). JAM-C and ESAM are involved in the promotion of melanoma lung metastasis formation (40).

Claudins are integral membrane proteins of the TJs that regulate the function of the TJs.

There are three members of the zonula occludens (ZO) family: ZO-1 (41), -2 (42) and -3. Common structural features of the $\mathrm{ZO}$ family include three PDZ domains in the N-terminal region, a SH3 (Src homology 3) domain and an enzymatically inactive guanylate kinase (GUK) domain. ZO proteins are important scaffold proteins, but are also essential in signaling processes (43-45). In addition, cingulin, AF-6 and 7H6 antigens are also important structural proteins of TJs. These peripheral proteins also play a role in maintaining the stability of TJs.

\section{Claudins}

Claudins were first identified by Furuse et al (41). In mammals, 24 members of the claudin-encoding gene family (CLDN genes 1-24) have been described: 23 in humans and chimpanzees and 24 in mice and rats. Claudins belong to the peripheral myelin protein (PMP22)/epithelial membrane protein (EMP) or membrane protein (MP20)/claudin superfamily of tetraspan membrane proteins (PFAM family 00822). Claudin genes are
22-34 kp in size with the majority of human claudin genes being 22-24 kp (42). The majority of claudins are located in epithelial and endothelial cells in all TJ-bearing tissues, however, a number of claudins have been observed in the cytoplasm $(46,47)$. The claudins in the cell membranes have four transmembranal helices, with their $\mathrm{NH}_{2}$ - and $\mathrm{COOH}$-terminal tails extending into the cytoplasm. A typical claudin protein contains a small intracellular cytoplasmic C-terminal sequence of $\sim 4-5$ residues, followed by a long extracellular loop of $\sim 24$ residues, a short 20-residue intracellular loop, another extracellular loop of $\sim 24$ residues and a $\mathrm{COOH}$-terminal cytoplasmic tail $(42,47)$. The amino acid sequences of the first and fourth transmembrane domains are highly conserved among claudins and the second and third are more diverse. The size of the carboxy-terminal cytoplasmic tail is most variable in length; it is typically between 21 and 63 residues. Different sections of claudin have different functions. The first extracellular loop contains highly conserved charged amino acids and is hypothesized to effect the formation of charge-selective channels in the paracellular space (48). Two highly conserved cysteines are expressed in the first extracellular loop of all claudins and potentially form an intramolecular disulfide bond to stabilize protein conformation (49). The exact function of the second extracellular loop remains unclear, however, it has been shown that claudin-5 in the second extracellular loop forms helix-turn-helix motifs with claudins on neighboring cell membranes, thus narrowing the paracellular cleft (50). The highly conserved residues Y148, Y158 and E159 in ECL2 of claudin-5 contribute to homo- and/or heterophilic trans-interaction between classic claudins and thereby tighten the paracellular space against ions, small and large molecules (51). The carboxy-terminal cytoplasmic tail of the claudins contains a PDZ-domain-binding motif that allows claudins to interact with cytoplasmic scaffolding proteins. Scaffolding proteins primarily include the TJ-associated proteins MUPP1 (52), Pals1-associated TJ protein (53), and ZO-1, -2 and -3 and membrane-associated guanylate kinase-like homologues (54). Furthermore, the $\mathrm{COOH}$-terminal tail upstream of the PDZ-binding motif is required to target to the tight junctional complex and also acts as a determinant of protein stability and function $(55,56)$.

Interactions between claudins may be homo- and heterotypic. It has been suggested that the primary structure of claudin-based paracellular pores may be formed via homotypic interactions $(57,58)$. There are two subsets of heterotypic interactions: between claudins of the same cell membrane (side-by-side interaction) and between claudins of opposing cell membranes (head-to-head interaction). Side-by-side and head-to-head interactions are limited to specific combinations of claudins (59-62).

Claudin function is typically evaluated by sampling gene expression at a number of points corresponding to gene knock-out or overexpression in the vast majority of studies. However, knowledge of the exact function of each type of claudins remains incomplete. It has been demonstrated that claudins have a close connection with embryonic morphogenesis and abnormal expression of claudins is markedly linked to various diseases, including malignant tumours. For example, abnormal expression of claudin-1, -6 and -7 has been observed in a number of skin diseases; claudin- $2,-5$ and -8 are associated 
with the gastrointestinal system; and abnormality in claudin-9, -11 and -14 is associated with hearing impairment $(42,63)$.

Distribution of claudins varies in different tissues. Claudin-5 is the dominant claudin in BBB endothelial cells, although claudins-1, -3 and -12 are also expressed in these cells (64-66). Claudin- 5 has been described as being the key factor involved in the endothelial permeability of the $\operatorname{BBB}(67,68)$.

\section{Claudin-5 plays a role in the process of $\mathrm{BM}$ via the regulation of $\mathrm{BBB}$ permeability}

Basic structure. Claudin-5 deficiency was first described by Morita et al (69) in patients with velo-cardio-facial syndrome hereditary disease. Claudin-5 is a protein encoded by the CLDN5 gene which contains only one intron and has two transcript variants (42). It contains 218 amino acids, with a molecular weight of 23,145 Da. As it has typical molecular structure of a claudin, it is hypothesized to function as a typical claudin.

Distribution. According to Morita et al (69), claudin-5 is an endothelial-specific component in the brain and lung vasculatures. Morita et al and Rahner et al also demonstrated that claudin-5 is expressed in the liver ECs and dermal vascular endothelia $(70,71)$. However, claudin-5 expression has been observed in uterine epithelial cells in the uterus of pregnant/gravid squamate reptiles and HT-29/B6 cells, an epithelial cell line derived from the human colon $(72,73)$. It has been confirmed that the principal claudin in BBB is claudin-5 and that it is an endothelial-specific component of the cell membrane of BBB (64-66,74), suggesting that claudin-5 is important in BBB.

Function of claudin-5. Claudin-5 is observed to be a key component of the TJ strand, particularly in brain endothelial cells. The major role of claudin-5 is to selectively decrease the permeability to ions. In particular, the conserved cysteines are crucial: mutation of either cysteine eliminates the ability of claudin-5 to increase transepithelial resistance (75). The $\mathrm{COOH}$-terminal tail of claudin-5 interacts with scaffolding proteins and is required for the apical localization at TJs (55). The function of TJs largely relies on homo- and heterophilic trans-interactions of claudin-5 and other claudins. Hemophilic trans-interaction is the interaction between claudin- 5 proteins and heteropolymers may be formed by claudins-1, -3 and -5 (76). Claudin-3 and -5 form elliptic meshes to restrict macromolecules passing through the tissue barrier. Two populations of elliptic meshes, with a mean diameter of $<100$ and 300-600 nm, respectively, have been observed. This function of claudin- 5 is not exclusive to BBB. It has been reported that claudin-5 expression and junctional organization controls human dermal microvascular ECs and arteriolar-capillary paracellular barriers. The barrier includes transendothelial electric resistance and macromolecular flux (77).

The role of claudin-5 in TJs has attracted attention over the past two decades. In a number of pathological processes, including inflammation, oedema, toxic damage, trauma and tumour, claudin-5 and any regulating factor have been observed to mediate the change in endothelial or epithelial permeability. It was demonstrated that matrix metalloproteinases (MMPs) open the BBB by degrading TJ proteins, claudin-5 and occludin and increasing BBB permeability following stroke. Additionally, an MMP inhibitor prevents degradation of TJ proteins and attenuates BBB disruption (78). Exposure of brain microvascular endothelial cells to high glucose increased BBB permeability in parallel with reduced expression levels of claudin- 5 and also confirmed that claudin- 5 is a key determinant of BBB permeability (79). Similarly, exposure to neurotoxicants malathion and lead acetate induces increased BBB permeability with decreased protein levels of TJ proteins, including claudin-5 (80). According to an in vitro BBB model, culture $\mathrm{pH}$ and buffer concentration have a significant impact on BBB permeability. This regulation may be mediated by increased claudin-5 expression (81). These data indicate that claudin-5 is important in the regulation of BBB permeability by modifying TJs.

The function of claudin-5 is regulated by a number of factors. Cyclic AMP (cAMP) was observed to elevate the barrier function of TJs in porcine BBB endothelial cells. This elevation is achieved through the protein kinase $A$ (PKA)-induced phosphorylation of claudin-5 immuno-precipitates or via the PKA-independent induction of claudin-5 (82). Similarly, in rat lung endothelial cells, it was also observed that claudin-5 expression is required to elevate endothelial barrier functions in response to cAMP (83). ERG plays a pivotal role in regulating EC barrier function and this effect is mediated, in part, through its regulation of CLDN5 gene expression (84). Transforming growth factor- $\beta 1$ increases the tyrosine phosphorylation of VE-cadherin and claudin-5. This process is involved in the increased paracellular permeability of CNS-derived vascular endothelium (85). The correlation between claudin- 5 and other cells in the BBB has also been studied. It was observed that glial cell line-derived neurotrophic factor secreted from pericytes increases the expression of claudin-5 and the TEER of brain microvascular endothelial cell, thus increasing the barrier function of the BBB (86). HIV-1 Tat protein contributes to alterations of the expression of claudin-5 and other TJs-associated proteins through activation of vascular endothelial growth factor receptor-2 and multiple redox-regulated signal transduction pathways $(87,88)$. Other factors, including sodium caprate, $\beta 1$-integrin and bradykinin are also involved in the regulation of claudin-5 (89-91).

Claudin-5 and BM. It has been hypothesized that the peritumoural brain edema in glioblastoma multiforme is a result of the downregulation of claudin-1 and -5 and occludin expression (92). One of the characteristics of a metastatic tumour is significant peritumoural edema, which may be explained as a result of the alteration in TJs and claudin-5. In superficial oesophageal squamous cell carcinoma, lymph node metastasis was observed to be associated with claudin-5 expression, although the exact mechanism is undetermined (93).

In a study of claudin-5 deficient mice, the ability of the BBB to act against small molecules $(<800 \mathrm{Da})$, but not larger molecules, was selectively reduced (68). This size-selective loosening of the BBB supports the hypothesis that claudin-5 is important in selective regulation of $\mathrm{BBB}$ permeability, which is a determinant of tumour metastasis. Furthermore, evidence exists to support the hypothesis that claudin promotes the activation of pro-MMP-2 (94). Caveolae-dependent internalization/recycling of claudin-5 was observed to transiently increase 
brain endothelial paracellular permeability during CNS inflammation $(95,96)$. In addition, claudin-5 was demonstrated to regulate endothelial motility. Escudero-Esparza et al (97) inserted claudin-5 into a human vascular endothelial cell line and noted a significant downregulation of motility, adhesive to matrix and angiogenic potential of vascular endothelial cells, indicating that claudin-5 may function through N-WASP and ROCK signaling pathways. A similar phenomenon was observed in breast cancer. Findings of those studies suggest that claudin-5 alters the biological behavior of tumour cells and plays a role in the formation of tumour metastases. In addition, it was observed in human immunodeficiency virus-1 encephalitis, that Rho kinase directly induces phosphorylation of occludin and claudin-5, resulting in decreased barrier tightness and enhanced monocyte migration across the BBB $(98,99)$. This mechanism may provide an explanation as to the increased permeability of $\mathrm{BBB}$ and cell migration across the BBB. Whether brain metastases follow the same mechanism remains unknown.

\section{Conclusion and future prospects}

Intracranial metastatic tumours have a higher incidence compared with primary intracranial tumours. BM involves a number of steps. A vital step is the transmigration of detached primary tumour cells through the BBB into the brain parenchyma. The BBB comprises endothelial cells, perticytes and astrocytes. These components play various roles in the $\mathrm{BBB}$, making the $\mathrm{BBB}$ a selective barrier. TJs constitute the main structure of the paracellular barrier between endothelial cells and claudins and are key proteins regulating permeability of TJs. The principle claudin in BBB is claudin-5. This claudin has two primary functions: regulation of $\mathrm{BBB}$ permeability and regulation of cell motility. These two functions are involved in the mechanism of brain metastases. Therefore, the association between claudin-5 and brain metastases is of great interest. Two issues remain to be resolved: how the primary tumour cells affect the vital claudins (claudin-5) in the TJs of brain microvascular endothelial cells and result in increased BBB permeability and BM and through which mechanism the claudins (claudin-5) regulate the motility, adhesion to the matrix and angiogenic potential of the tumour cells to complete their transportation.

The role of the BBB in brain metastases has attracted great interest. A number of studies have been performed on transmembrane proteins, including claudins and occludins. However, the majority of studies focus on the correlation between the changes in TJ proteins in carcinoma cells and enhancement of invasion ability of the tumour. A number of investigators have considered the interaction between tumour cells and endothelial cells. However, the endothelial cell lines used, such as HECV, in these studies are derived from the umbilical vein system, not from brain microvascular endothelial cell lines, thus they do not represent ECs in the BBB. Claudin-5, as a distinct and important transmembrane protein of BBB TJs, is confirmed to be involved in the process of tumour cell migration into the brain through paracellular passage. However, little is currently known of the regulation of claudin-5 on BBB TJs and the signaling pathways involved in BM. It is clear that this area requires further investigation.

\section{Acknowledgements}

The authors would like to thank Cancer Research Wales and the Albert Hung Foundation for supporting their study. Dr W Jia is a recipient of the China Medical Scholarship of Cardiff University.

\section{References}

1. Wilhelm I, Molnár J, Fazakas C, Haskó J and Krizbai IA: Role of the blood-brain barrier in the formation of brain metastases. Int J Mol Sci 14: 1383-1411, 2013

2. Walker AE, Robins M and Weinfeld FD: Epidemiology of brain tumors: the national survey of intracranial neoplasms. Neurology 35: 219-226, 1985

3. Gavrilovic IT and Posner JB: Brain metastases: epidemiology and pathophysiology. J Neurooncol 75: 5-14, 2005.

4. Schouten LJ, Rutten J, Huveneers HA and Twijnstra A: Incidence of brain metastases in a cohort of patients with carcinoma of the breast, colon, kidney, and lung and melanoma. Cancer 94: 2698-2705, 2002

5. Preusser M, Capper D, Ilhan-Mutlu A, et al: Brain metastases: pathobiology and emerging targeted therapies. Acta Neuropathol 123: 205-222, 2012.

6. Barnholtz-Sloan JS, Sloan AE, Davis FG, Vigneau FD, Lai P and Sawaya RE: Incidence proportions of brain metastases in patients diagnosed (1973 to 2001) in the Metropolitan Detroit Cancer Surveillance System. J Clin Oncol 22: 2865-2872, 2004.

7. Platta CS, Khuntia D, Mehta MP and Suh JH: Current treatment strategies for brain metastasis and complications from therapeutic techniques: a review of current literature. Am J Clin Oncol 33: 398-407, 2010.

8. Lim LC, Rosenthal MA, Maartens N and Ryan G: Management of brain metastases. Intern Med J 34: 270-278, 2004.

9. Lu-Emerson C and Eichler AF: Brain metastases. Continuum (Minneap Minn) 18: 295-311, 2012.

10. Delattre JY, Krol G, Thaler HT and Posner JB: Distribution of brain metastases. Arch Neurol 45: 741-744, 1988.

11. Gaspar LE, Mehta MP, Patchell RA, et al: The role of whole brain radiation therapy in the management of newly diagnosed brain metastases: a systematic review and evidence-based clinical practice guideline. J Neurooncol 96: 17-32, 2010.

12. Linskey ME, Andrews DW, Asher AL, et al: The role of stereotactic radiosurgery in the management of patients with newly diagnosed brain metastases: a systematic review and evidence-based clinical practice guideline. J Neurooncol 96: 45-68, 2010.

13. Mehta MP, Paleologos NA, Mikkelsen T, et al: The role of chemotherapy in the management of newly diagnosed brain metastases: a systematic review and evidence-based clinical practice guideline. J Neurooncol 96: 71-83, 2010.

14. Kalkanis SN, Kondziolka D, Gaspar LE, et al: The role of surgical resection in the management of newly diagnosed brain metastases: a systematic review and evidence-based clinical practice guideline. J Neurooncol 96: 33-43, 2010.

15. Olson JJ, Paleologos NA, Gaspar LE, et al: The role of emerging and investigational therapies for metastatic brain tumors: a systematic review and evidence-based clinical practice guideline of selected topics. J Neurooncol 96: 115-142, 2010.

16. Fokas E, Steinbach JP and Rödel C: Biology of brain metastases and novel targeted therapies: time to translate the research. Biochim Biophys Acta 1835: 61-75, 2013.

17. Correale J and Villa A: Cellular elements of the blood-brain barrier. Neurochem Res 34: 2067-2077, 2009.

18. Bearer EL and Orci L: Endothelial fenestral diaphragms: a quick-freeze, deep-etch study. J Cell Biol 100: 418-428, 1985.

19. Brightman MW and Reese TS: Junctions between intimately apposed cell membranes in the vertebrate brain. J Cell Biol 40: 648-677, 1969

20. Lane NJ, Reese TS and Kachar B: Structural domains of the tight junctional intramembrane fibrils. Tissue Cell 24: 291-300, 1992.

21. Villegas JC and Broadwell RD: Transcytosis of protein through the mammalian cerebral epithelium and endothelium. II Adsorptive transcytosis of WGA-HRP and the blood-brain and brain-blood barriers. J Neurocytol 22: 67-80, 1993. 
22. Dorovini-Zis K, Prameya R and Bowman PD: Culture and characterization of microvascular endothelial cells derived from human brain. Lab Invest 64: 425-436, 1991.

23. Armulik A, Abramsson A and Betsholtz C: Endothelial/pericyte interactions. Circ Res 97: 512-523, 2005.

24. Winkler EA, Bell RD and Zlokovic BV: Central nervous system pericytes in health and disease. Nat Neurosci 14: 1398-1405, 2011.

25. Dalkara T, Gursoy-Ozdemir Y and Yemisci M: Brain microvascular pericytes in health and disease. Acta Neuropathol 122 $1-9,2011$.

26. Kacem K, Lacombe P, Seylaz J and Bonvento G: Structural organization of the perivascular astrocyte endfeet and their relationship with the endothelial glucose transporter: a confocal microscopy study. Glia 23: 1-10, 1998.

27. Cohen Z, Molinatti G and Hamel E: Astroglial and vascular interactions of noradrenaline terminals in the rat cerebral cortex. J Cereb Blood Flow Metab 17: 894-904, 1997.

28. Paspalas CD and Papadopoulos GC: Ultrastructural relationships between noradrenergic nerve fibers and non-neuronal elements in the rat cerebral cortex. Glia 17: 133-146, 1996

29. Zhao YN, Wang F, Fan YX, Ping GF, Yang JY and Wu CF: Activated microglia are implicated in cognitive deficits, neuronal death, and successful recovery following intermittent ethanol exposure. Behav Brain Res 236: 270-282, 2013.

30. Readnower RD, Chavko M, Adeeb S, et al: Increase in blood-brain barrier permeability, oxidative stress, and activated microglia in a rat model of blast-induced traumatic brain injury. J Neurosci Res 88: 3530-3539, 2010.

31. Rascher G, Fischmann A, Kröger S, Duffner F, Grote EH and Wolburg H: Extracellular matrix and the blood-brain barrier in glioblastoma multiforme: spatial segregation of tenascin and agrin. Acta Neuropathol 104: 85-91, 2002.

32. Jian Liu K and Rosenberg GA: Matrix metalloproteinases and free radicals in cerebral ischemia. Free Radic Biol Med 39: 71-80, 2005

33. Abbott NJ, Patabendige AA, Dolman DE, Yusof SR and Begley DJ: Structure and function of the blood-brain barrier. Neurobiol Dis 37: 13-25, 2010.

34. Wilhelm I, Fazakas $C$ and Krizbai IA: In vitro models of the blood-brain barrier. Acta Neurobiol Exp (Wars) 71: 113-128, 2011.

35. Furuse M, Hirase T, Itoh M, Nagafuchi A, Yonemura S, Tsukita S and Tsukita S: Occludin: a novel integral membrane protein localizing at tight junctions. J Cell Biol 123: 1777-1788, 1993

36. Liu WY, Wang ZB, Zhang LC, Wei X and Li L: Tight junction in blood-brain barrier: an overview of structure, regulation, and regulator substances. CNS Neurosci Ther 18: 609-615, 2012.

37. Luissint AC, Artus C, Glacial F, Ganeshamoorthy K and Couraud PO: Tight junctions at the blood brain barrier: physiological architecture and disease-associated dysregulation. Fluids Barriers CNS 9: 23, 2012.

38. Feldman GJ, Mullin JM and Ryan MP: Occludin: structure, function and regulation. Adv Drug Deliv Rev 57: 883-917, 2005.

39. Förster C: Tight junctions and the modulation of barrier function in disease. Histochem Cell Biol 130: 55-70, 2008.

40. Langer HF, Orlova VV, Xie C, et al: A novel function of junctional adhesion molecule- $\mathrm{C}$ in mediating melanoma cell metastasis. Cancer Res 71: 4096-4105, 2011

41. Furuse M, Fujita K, Hiiragi T, Fujimoto $K$ and Tsukita $S$ : Claudin-1 and -2: novel integral membrane proteins localizing at tight junctions with no sequence similarity to occludin. J Cell Biol 141: 1539-1550, 1998.

42. Lal-Nag M and Morin PJ: The claudins. Genome Biol 10: 235 , 2009.

43. Gumbiner B, Lowenkopf $\mathrm{T}$ and Apatira D: Identification of a $160-\mathrm{kDa}$ polypeptide that binds to the tight junction protein ZO-1. Proc Natl Acad Sci USA 88: 3460-3464, 1991.

44. Stevenson BR, Siliciano JD, Mooseker MS and Goodenough DA: Identification of ZO-1: a high molecular weight polypeptide associated with the tight junction (zonula occludens) in a variety of epithelia. J Cell Biol 103: 755-766, 1986.

45. Haskins J, Gu L, Wittchen ES, Hibbard J and Stevenson BR $\mathrm{ZO}-3$, a novel member of the MAGUK protein family found at the tight junction, interacts with $\mathrm{ZO}-1$ and occludin. J Cell Biol 141: 199-208, 1998.

46. Blackman B, Russell T, Nordeen SK, Medina D and Neville MC: Claudin 7 expression and localization in the normal murine mammary gland and murine mammary tumors. Breast Cancer Res 7: R248-R255, 2005.
47. Morin PJ: Claudin proteins in human cancer: promising new targets for diagnosis and therapy. Cancer Res 65: 9603-9606, 2005.

48. Colegio OR, Van Itallie CM, McCrea HJ, Rahner C and Anderson JM: Claudins create charge-selective channels in the paracellular pathway between epithelial cells. Am J Physiol Cell Physiol 283: C142-C147, 2002.

49. Angelow S, Ahlstrom R and Yu AS: Biology of claudins. Am J Physiol Renal Physiol 295: F867-F876, 2008.

50. Piontek J, Winkler L, Wolburg H, et al: Formation of tight junction: determinants of homophilic interaction between classic claudins. FASEB J 22: 146-158, 2008

51. Piehl C, Piontek J, Cording J, Wolburg H and Blasig IE: Participation of the second extracellular loop of claudin-5 in paracellular tightening against ions, small and large molecules. Cell Mol Life Sci 67: 2131-2140, 2010.

52. Hamazaki Y, Itoh M, Sasaki H, Furuse M and Tsukita S: Multi-PDZ domain protein 1 (MUPP1) is concentrated at tight junctions through its possible interaction with claudin-1 and junctional adhesion molecule. J Biol Chem 277: 455-461, 2002.

53. Roh MH, Liu CJ, Laurinec S and Margolis B: The carboxyl terminus of zona occludens-3 binds and recruits a mammalian homologue of discs lost to tight junctions. J Biol Chem 277: 27501-27509, 2002.

54. Itoh M, Furuse M, Morita K, Kubota K, Saitou M and Tsukita S Direct binding of three tight junction-associated MAGUKs, $\mathrm{ZO}-1, \mathrm{ZO}-2$, and $\mathrm{ZO}-3$, with the $\mathrm{COOH}$ termini of claudins. J Cell Biol 147: 1351-1363, 1999.

55. Rüffer C and Gerke V: The C-terminal cytoplasmic tail of claudins 1 and 5 but not its PDZ-binding motif is required for apical localization at epithelial and endothelial tight junctions. Eur J Cell Biol 83: 135-144, 2004.

56. Van Itallie CM, Colegio OR and Anderson JM: The cytoplasmic tails of claudins can influence tight junction barrier properties through effects on protein stability. J Membr Biol 199: 29-38, 2004.

57. Mitic LL, Unger VM and Anderson JM: Expression, solubilization, and biochemical characterization of the tight junction transmembrane protein claudin-4. Protein Sci 12: 218-227, 2003.

58. Blasig IE, Winkler L, Lassowski B, et al: On the self-association potential of transmembrane tight junction proteins. Cell Mol Life Sci 63: 505-514, 2006

59. Furuse M, Sasaki $\mathrm{H}$ and Tsukita S: Manner of interaction of heterogeneous claudin species within and between tight junction strands. J Cell Biol 147: 891-903, 1999.

60. Daugherty BL, Ward C, Smith T, Ritzenthaler JD and Koval M: Regulation of heterotypic claudin compatibility. J Biol Chem 282: 30005-30013, 2007.

61. Coyne CB, Gambling TM, Boucher RC, Carson JL and Johnson LG: Role of claudin interactions in airway tight junctional permeability. Am J Physiol Lung Cell Mol Physiol 285: L1166-L1178, 2003.

62. Ward C and Koval M: Biochemical analysis of claudin-binding compatibility. Methods Mol Biol 762: 13-26, 2011.

63. Gupta IR and Ryan AK: Claudins: unlocking the code to tight junction function during embryogenesis and in disease. Clin Genet 77: 314-325, 2010

64. Cooper I, Cohen-Kashi-Malina K and Teichberg VI: Claudin-5 expression in in vitro models of the blood-brain barrier. Methods Mol Biol 762: 347-354, 2011.

65. Neuhaus W, Wirth M, Plattner VE, Germann B, Gabor F and Noe CR: Expression of Claudin-1, Claudin-3 and Claudin-5 in human blood-brain barrier mimicking cell line ECV304 is inducible by glioma-conditioned media. Neurosci Lett 446 : 59-64, 2008

66. Ohtsuki S, Yamaguchi H, Katsukura Y, Asashima T and Terasaki T: mRNA expression levels of tight junction protein genes in mouse brain capillary endothelial cells highly purified by magnetic cell sorting. J Neurochem 104: 147-154, 2008.

67. Ohtsuki S, Sato S, Yamaguchi H, Kamoi M, Asashima T and Terasaki T: Exogenous expression of claudin-5 induces barrier properties in cultured rat brain capillary endothelial cells. J Cell Physiol 210: 81-86, 2007.

68. Nitta T, Hata M, Gotoh S, et al: Size-selective loosening of the blood-brain barrier in claudin-5-deficient mice. J Cell Biol 161: 653-660, 2003.

69. Morita K, Sasaki H, Furuse M and Tsukita S: Endothelial claudin: claudin-5/TMVCF constitutes tight junction strands in endothelial cells. J Cell Biol 147: 185-194, 1999. 
70. Morita K, Sasaki H, Furuse K, Furuse M, Tsukita S and Miyachi Y: Expression of claudin-5 in dermal vascular endothelia. Exp Dermatol 12: 289-295, 2003.

71. Rahner C, Mitic LL and Anderson JM: Heterogeneity in expression and subcellular localization of claudins $2,3,4$, and 5 in the rat liver, pancreas, and gut. Gastroenterology 120: 411-422, 2001.

72. Biazik JM, Thompson MB and Murphy CR: Claudin-5 is restricted to the tight junction region of uterine epithelial cells in the uterus of pregnant/gravid squamate reptiles. Anat Rec (Hoboken) 291: 547-556, 2008.

73. Amasheh S, Schmidt T, Mahn M, et al: Contribution of claudin-5 to barrier properties in tight junctions of epithelial cells. Cell Tissue Res 321: 89-96, 2005.

74. Ohtsuki S, Ikeda C, Uchida Y, et al: Quantitative targeted absolute proteomic analysis of transporters, receptors and junction proteins for validation of human cerebral microvascular endothelial cell line hCMEC/D3 as a human blood-brain barrier model. Mol Pharm 10: 289-296, 2013.

75. Wen H, Watry DD, Marcondes MC and Fox HS: Selective decrease in paracellular conductance of tight junctions: role of the first extracellular domain of claudin-5. Mol Cell Biol 24 8408-8417, 2004.

76. Piontek J, Fritzsche S, Cording J, et al: Elucidating the principles of the molecular organization of heteropolymeric tight junction strands. Cell Mol Life Sci 68: 3903-3918, 2011.

77. Kluger MS, Clark PR, Tellides G, Gerke V and Pober JS Claudin-5 controls intercellular barriers of human dermal microvascular but not human umbilical vein endothelial cells Arterioscler Thromb Vasc Biol 33: 489-500, 2013.

78. Yang Y and Rosenberg GA: MMP-mediated disruption of claudin-5 in the blood-brain barrier of rat brain after cerebral ischemia. Methods Mol Biol 762: 333-345, 2011.

79. Liu C, Wu J and Zou MH: Activation of AMP-activated protein kinase alleviates high-glucose-induced dysfunction of brain microvascular endothelial cell tight-junction dynamics. Free Radic Biol Med 53: 1213-1221, 2012.

80. Balbuena P, Li W and Ehrich M: Assessments of tight junction proteins occludin, claudin 5 and scaffold proteins ZO1 and ZO2 in endothelial cells of the rat blood-brain barrier: cellular responses to neurotoxicants malathion and lead acetate. Neurotoxicology 32: 58-67, 2011.

81. Helms HC, Waagepetersen HS, Nielsen CU and Brodin B: Paracellular tightness and claudin-5 expression is increased in the BCEC/astrocyte blood-brain barrier model by increasing media buffer capacity during growth. AAPS J 12: 759-770, 2010.

82. Ishizaki T, Chiba H, Kojima T, et al: Cyclic AMP induces phosphorylation of claudin-5 immunoprecipitates and expression of claudin-5 gene in blood-brain-barrier endothelial cells via protein kinase A-dependent and -independent pathways. Exp Cell Res 290: 275-288, 2003.

83. Soma T, Chiba H, Kato-Mori Y, et al: Thr(207) of claudin-5 is involved in size-selective loosening of the endothelial barrier by cyclic AMP. Exp Cell Res 300: 202-212, 2004.

84. Yuan L, Le Bras A, Sacharidou A, et al: ETS-related gene (ERG) controls endothelial cell permeability via transcriptional regulation of the claudin 5 (CLDN5) gene. J Biol Chem 287: 6582-6591, 2012.
85. Shen W, Li S, Chung SH, et al: Tyrosine phosphorylation of VE-cadherin and claudin-5 is associated with TGF- $\beta 1$-induced permeability of centrally derived vascular endothelium. Eur J Cell Biol 90: 323-332, 2011

86. Shimizu F, Sano Y, Saito K, et al: Pericyte-derived glial cell line-derived neurotrophic factor increase the expression of claudin-5 in the blood-brain barrier and the blood-nerve barrier. Neurochem Res 37: 401-409, 2012.

87. András IE and Toborek M: HIV-1-induced alterations of claudin-5 expression at the blood-brain barrier level. Methods Mol Biol 762: 355-370, 2011.

88. András IE, Pu H, Tian J, et al: Signaling mechanisms of HIV-1 Tat-induced alterations of claudin-5 expression in brain endothelial cells. J Cereb Blood Flow Metab 25: 1159-1170, 2005.

89. Liu LB, Xue YX, Liu YH and Wang YB: Bradykinin increases blood-tumor barrier permeability by down-regulating the expression levels of ZO-1, occludin, and claudin-5 and rearranging actin cytoskeleton. J Neurosci Res 86: 1153-1168, 2008.

90. Osada T, Gu YH, Kanazawa M, et al: Interendothelial claudin-5 expression depends on cerebral endothelial cell-matrix adhesion by $\beta(1)$-integrins. J Cereb Blood Flow Metab 31: 1972-1985, 2011.

91. Del Vecchio G, Tscheik C, Tenz K, et al: Sodium caprate transiently opens claudin-5-containing barriers at tight junctions of epithelial and endothelial cells. Mol Pharm 9: 2523-2533, 2012.

92. Liebner S, Fischmann A, Rascher G, et al: Claudin-1 and claudin-5 expression and tight junction morphology are altered in blood vessels of human glioblastoma multiforme. Acta Neuropathol 100: 323-331, 2000

93. Chiba T, Kawachi H, Kawano T, et al: Independent histological risk factors for lymph node metastasis of superficial esophageal squamous cell carcinoma; implication of claudin-5 immunohistochemistry for expanding the indications of endoscopic resection. Dis Esophagus 23: 398-407, 2010.

94. Miyamori H, Takino T, Kobayashi Y, et al: Claudin promotes activation of pro-matrix metalloproteinase- 2 mediated by membrane-type matrix metalloproteinases. J Biol Chem 276: 28204-28211, 2001.

95. Stamatovic SM, Keep RF, Wang MM, Jankovic I and Andjelkovic AV: Caveolae-mediated internalization of occludin and claudin-5 during CCL2-induced tight junction remodeling in brain endothelial cells. J Biol Chem 284: 19053-19066, 2009.

96. Stamatovic SM, Keep RF and Andjelkovic AV: Tracing the endocytosis of claudin-5 in brain endothelial cells. Methods Mol Biol 762: 303-320, 2011

97. Escudero-Esparza A, Jiang WG and Martin TA: Claudin-5 is involved in breast cancer cell motility through the N-WASP and ROCK signalling pathways. J Exp Clin Cancer Res 31: 43, 2012.

98. Yamamoto M, Ramirez SH, Sato S, et al: Phosphorylation of claudin-5 and occludin by rho kinase in brain endothelial cells. Am J Pathol 172: 521-533, 2008.

99. Persidsky Y, Heilman D, Haorah J, et al: Rho-mediated regulation of tight junctions during monocyte migration across the blood-brain barrier in HIV-1 encephalitis (HIVE). Blood 107: 4770-4780, 2006. 